[12] Poroher Oh. et Maynard L. 1930. La graisse du sang et la graisse du lait pendant la lactation. Le Lait, vol. 10, p. 601-613 et 765-782.

[13] Roodzant. 1931. Onderzockingen betreffende de correlatie tussehen het vetgehalte van de Melk en het lipoïdenhalt van het bloed. Dissert. Utrecht.

[14] SjoLlima. 1923. Voeding an Melkproductie. Culture.

[15] SMorodtnzerry. 1929. Le cholestérol et son importance en physiologie et en pathologie. "Ouspecloie biologuitschesloi Chimii ", vol. VII, p. 104-135 (russe).

\title{
LA MODIFICATION DE LA COMIPOSITION DU LAIT A L'ÉPOQUE DE LA PAISSON
}

\section{par ZOLTAN CSUKAS}

Docteur vétérinaire et docteur agr., professeur adjoint d'université.

La science recherche depuis longtemps déjà dans quelle. mesure la paisson influence la composition du lait, et particulièrement sa teneur en graisse. Si la modification de la eomposition n'atteignait que des dixièmes de pourcentage, on pourrait trouver l'explication dans les conditions vitales variant quotidiennement et dont l'importance théorique et pratique est restreinte. Selon la littérature, cependant, la teneur en graisse du lait, à l'époque en question, peut tomber au-dessous de $2 \%$ ou monter au-dessus de $4,5 \%$, même dans une moyenne "d'étable».

L'observation des conditions contradictoires complique la = eomparaison des données écrites (HITTOHER et GRIMMER, BÜNGER et Altmann, Kirsten, Olmmanns, Sommerfeld, Stegmann, Thikötter, Amschler, Chrzaszoz, Cambell, Hills, Regsdale et Turner, Brody, Drakeley et White, Popoff, Housten et HaLE, etc.). Les données américaines, danoises, anglaises et, en partie, allemandes ont été obtenues sous des climats très variés, ce qui suffit à expliquer les résultats contradictoires. Dans les expériences d'étable (où les autres facteurs ont été éliminés), la corrélation néga tive entre la température ascendante et la teneur en graisse a aussi été constatée de façon unanime. Le contraire, cependant, a été observé dans les expériences de pâturage où le rôle alternatif de la variation de température ne s'est montré dans aucune direction.

Au cours de mes expériences, en 1932 et 1933, j'ai examiné dans les semaines précédant la pousse de l'herbe et pendant le mois suivant celle-ci, le lait de 140 vaches provenant de six exploitations agricoles différentes. En voici le résultat :

Dans les premières semaines de la paisson, la teneur en graisse du lait et en extrait sec dégraissé, change ainsi que la flore microbienne du pis. La cause de l'importance et du sens de la modification doit être cherchée dans les influences extérieures. 
Plus il y a de facteurs et plus grande est la mesure dans laquelle ils troublent les phénomènes vitaux, plus l'hérédité et le milieu ont donné à l'organisme une ca pacité de réaction et plus la modification est grande.

Avec la réduction des influences extérieures et par l'adaptation de l'organisme, l'écart du normal de la teneur du lait en graisse, en extrait sec dégraissé, à l'époque de la paisson, diminue généralement dans une mesure dépassant $0,5 \%$, puis augmente dans les 4 ¿े 20 jours de plus de $0,5 \%$. Au cours des premières semaines de la paisson, la modification individuelle en ce qui concerne la teneur en graisse, excède de deux à trois fois la fluctuation normale.

L'influence de la paisson ne fait pas non plus varier journellement dans une même direction la teneur en graisse du lait d'un même individu. Les individus possédant un organisme fin réagissent davantage sous l'influence des facteurs du milieu agissant au début de la paisson.

Il n'existe aucune corrélation entre le changement de la quantité du lait et sa teneur en graisse. La teneur en extrait sec dégraissé diminue d'abord après la pousse et augmente dans la suite. Cette substance varie à peine et seulement chez les individus dont la teneur en graisse du lait varie dans les limites normales.

La teneur en extrait sec dégraissé peut aussi tomber au-dessous de $8,5 \%$ dans la moyenne du cheptel et peut aussi permettre de soupçonner une falsification.

Dans les premières semaines de la paisson, les enzymes produites par les microcoques peuvent cailler le lait en 24 heures. Le caillage précoce du lait est plus fréquent chez les individus dont la teneur du lait en graisse et en extrait sec dégraissé a le plus varié. La cause immédiate du changement de la composition du lait est la diminution de la résistance de l'organisme, sur lequel l'affouragement et le climat ont la plus grande influence. Parmi les influences secondaires, les efforts physiques exigés par la paisson (l'envoi des vaches d'étable dans les pâturages lointains, le dérangement causé par les gardiens et les chiens) et la capacité de résistance de l'organisme ont réellement de l'importance.

L'affouragement précédent a aussi une influence incontestée. En effet, si au cours de la paisson, le processus vital, déjà troublé par d'autres causes, est encore perturbé par une modification subite dans l'affouragement, le changement de milieu se présente alors d'une manière plus marquée quant au fonctionnement des glandes sécrétrices de lait, dont l'échange organique est si vif.

Concernant l'effet spécifique de la flore, il y a lieu de savoir si l'herbe du pâturage ou le fourrage vert n'a pas remplacé tels éléments du mélange de fourrage sec, éléments qui, comme l'expérience 
nous l'a démontré jusqu'ici, augmentent ou diminuent la teneur en graisse du lait. On ne peut imputer au changement d'affouragement seul, la variation de la teneur en graisse, étant donné que : a) il n'a été constaté aucune augmentation de la teneur en graisse supérieure à $0,5 \%$ ou une diminution, quel qu'ait été le fourrage absorbé ; b) la teneur en graisse a augmenté ou diminué chez chaque individu par l'influence de la paisson, contrairement à ce qu'on devrait escompter par l'effet spécifique de la nourriture; $c$ ) la teneur en graisse a ou augmenté ou diminué chez le même individu, et, enfin, d) il a également été observé un changement identique dans le senș de la variation de la teneur en graisse après la paisson dans des pâturages soudeux, sablonneux et artificiels de composition diverse.

L'expérience prouve encore qu'on ne peut considérer l'affouragement vert comme indifférent, expérience selon laquelle la teneur en graisse a aussi baissé dans une partie des troupeaux confinés à l'étable, alors qu'une quantité abondante de fourrage vert avait remplacé celui d'hiver. La teneur en graisse a de même diminué avant la pousse dans le pâturage de deux domaines lorsque les vaches recevaient du fourrage vert.

Au cours de l'affouragement de fin d'hiver, la mauvaise proportion des matières minérales trouble le métabolisme du calcium chez les individus sécrétant particulièrement beaucoup de chaux par suite d'une fourniture abondante de lait. Si même la quantité et la proportion des matières minérales n'ont pas d'influence directe sur la composition du lait, il n'est point douteux que ces facteurs intensifient le trouble général de l'échange organique et diminuent la capacité de résistance de l'organisme. L'influence directe sur l'aptitude à la production est aussi incontestable. Je me réfère, quant à ce qui précède, à la coutume des paysans tendant à donner de la marne aux vaches le jour de la paisson, voyant en cette façon de faire un préventif de la "tétanie» causée par le pâturage (Grastetanie). Je noterai encore le fait que le lait produit dans des pâturages pauvres en matières minérales solubles, caille plus rapidement.

Mes propres expériences révèlent que les données écrites ne sont, en substance, nullement contradictoires, ear rien n'empêche de constater au début de la paisson l'augmentation ou la diminution de la teneur en graisse suivant la nature de l'influence qu'exercent les facteurs de l'affouragement et la façon d'être précédents, la technique de la paisson, la capacité de résistance modifiée par le milieu. Ces mêmes facteurs, aux combinaisons nombreuses, sont aussi la cause que la teneur en graisse du lait s'écarte de la valeur normale pendant des périodes très variables. La modification assez fréquente dans certaines provinces des éléments composants du lait, s'explique par le fait que, dans ces régions, la situation de 
l'affouragement et le "modus vivendi » à l'époque à laquelle commence la paisson, les vicissitudes du climat, les caractéristiques individuelles du cheptel élevé, ete., mettent davantage à l'épreuve et pour une durée plus longue la capacité de résistance.

L'explication classique pour la prévention de la modification de la composition du lait, ou mieux, son atténuation, offre d'ellemême les procédés généralement connus, assurant les phénomènes vitaux normaux.

Les données de l'expérience confirment que là où, en ce qui concerne le elimat, la quantité et la qualité du fourrage, la transition était graduelle au point de vue physique, les éléments examinés du lait n'ont atteint que temporairement et dans une faible mesure, un taux supérieur à celui de la valeur normale.

Etant donné que la teneur en extrait see dégraissé n'a pas paru constante, l'hypothèse que son examen pourrait prouver ou démentir la falsification ne s'est done pas vérifiée.

(Travail de la Faculté des sciences économiques de l'Institut zootechnique de Budapest. Directeur : Prof. J. SoHandL.)

\title{
CONSIDÉRATIONS SUR L'INFLUENCE DU PASSAGE DANS LE LAIT DE QUELQUES PRINCIPES D'ORIGINE MINÉRALE OU VÉGÉTALE
}

\author{
par M. LAGRANGE-FRANCÈS
}

Docteur en pharmacie.

Il est bien connu que nombre de médicaments passent dans le lait. Sigalas et Dupoux, notamment, ont montré que le lait des nourrices soumises à un traitement hydrargyrique contient du mercure; Dervieux, Szumlanski et Desvillees ont montré la présence d'alcool dans le lait d'une jeune aleoolique et le passage de ce composé dans les organes de son nourrisson de 4 mois, mort de broncho-pneumonie.

D'autre part, PoroHer a écrit :

"Si l'on tient pour fondé le passage des substances médicamenteuses dans le lait, la question mérite qu'on s'y arrête et cela pour deux raisons :

$1^{\circ} \mathrm{Qu}$ 'on veuille préparer des laits dits médicamenteux,

$2^{\circ}$ Que l'on tienne, dans un esprit cette fois tout opposé, à interdire, comme le fait le règlement de plusieurs villes allemandes, la vente du lait d'animaux ayant absorbé certains produits dont, à tort ou à raison, on redoute le passage dans le lait. "

D'autre part, beaucoup d'auteurs ont signalé les modifications organoleptiques du lait d'animaux a yant absorbé certains végétaux. 